\title{
Diabetic kidney disease and diabetic retinopathy: the ominous duo
}

\section{Vijay Viswanathan ${ }^{1}$}

Published online: 1 September 2020

(C) Research Society for Study of Diabetes in India 2020

Diabetic kidney disease (DKD) and diabetic retinopathy (DR) are two feared complications of diabetes. The article by Dash et al., in this journal, "Diabetic retinopathy and its association with low glomerular filtration rate: a cross sectional analysis of diabetes patients of community clinics across India", looks at the association between these two microvascular complications [1].

In this retrospective study of 1547 people with type 2 diabetes mellitus (T2DM), they looked at the association between DKD and DR in 443 people. The prevalence of DR was found to be more in people with lower estimated glomerular filtration rate (eGFR) of less than $60 \mathrm{ml} / \mathrm{min} / 1.73 \mathrm{~m}^{2}$. There was also a significant correlation between low levels of eGFR and DR [1].

The expenditures on hospitalisation for people with diabetes and DKD in India are considerably higher than in those without any complication [2]. In a 12-year observational study in India, Viswanathan et al. found that among 152 people who had normoalbuminuria at baseline, the presence of diabetic retinopathy showed a significant association with the development of macroalbuminuria [3]. In another study by the author, comparing people with proteinuria and those without proteinuria, it was observed that diabetic retinopathy was present in $100 \%$ of proteinuric patients vs $24 \%$ in the nonproteinuric group [4].

The author also studied the renal-retinal relationship in people with T2DM in India [5]. The mean decline in renal function worsened significantly as the severity of DR increased. The mortality rate was higher in people with both DKD and DR.

In a recent study done in a tertiary care diabetes centre in South India, the effect of DKD on the development of newonset DR and sight-threatening diabetic retinopathy (STDR)

Vijay Viswanathan

drvijay@mvdiabetes.com

1 Prof M Viswanathan Diabetes Research Centre, Chennai, India was studied [6]. Higher serum creatinine, low eGFR and the presence of macroalbuminuria were associated with increased risk of progression to STDR [6].

From the above-mentioned discussion, it is very evident that in people with DM, the presence of DR is associated with worsening kidney function. It is also seen that in people with declining kidney function, the risk of severe DR is also increased. The relationship between the degree of diabetic retinopathy and the severity of glomerular lesions in people with T2DM may not always show concordance.

Discordance between DKD and DR occurs often. Some people with DR do not have any evidence of DKD. Discordance between these two conditions is believed to be due to some differences in the pathogenesis of these two conditions. A recent population-based study on multi-ethnic Asian adults found that those patients with concordance of both DKD and DR had a higher risk of all-cause and CV mortality [7].

The general concept is that all people with DKD will exhibit some degree of DR. The rapid onset or progression of proteinuria, lack of DR, presence of hematuria and active urine sedimentation suggest a non-diabetic etiology and warrant a kidney biopsy. It is also known that there exists an entity called normoalbuminuric DKD where chronic kidney disease is present without any albuminuria [8].

Therefore, it is mandatory to closely follow the kidney function in people with diabetes with both estimation of albumin in the urine and estimation of eGFR. The authors had shown that in India the CKD Epi $[\mathrm{Sr} \mathrm{Cr}]$ is a good formula to determine the eGFR $[9,10]$. It is also important to motivate people with diabetes to have a regular eye test to diagnose common problems like cataract, glaucoma and DR.

Therefore in conclusion, every physician dealing with people with diabetes should educate their patients about the importance of regular screening for DR to prevent blindness and estimation of the urine albumin concentration by a simple urine albumin/creatinine ratio and calculation of the eGFR. 


\section{References}

1. Dash K, Ahmed A, Das S, Jaganmohan B, Tippisetty S, Kolukula VK, et al. Diabetic retinopathy and its association with low glomerular filtration rate: a cross-sectional analysis of diabetes patients of community clinics across India. Int J Diabetes Dev Ctries. 2020. https://doi.org/10.1007/s13410-019-00779-2.

2. Satyavani K, Kothandan H, Jayaraman M, Viswanathan V. Direct costs associated with chronic kidney disease among type 2 diabetic patients in India. Indian J Nephrol. 2014;24(3):141-7. https://doi. org/10.4103/0971-4065.132000.

3. Viswanathan V, Tilak P, Kumpatla S. Risk factors associated with the development of overt nephropathy in type 2 diabetes patients: a 12 years observational study. Indian J Med Res. 2012;136(1): 46-53.

4. Viswanathan V, Snehalatha C, Ramachandran A, Viswanathan M. Proteinuria in NIDDM in South India: analysis of predictive factors. Diabetes Res Clin Pract. 1995;28(1):41-6. https://doi.org/10.1016/ 0168-8227(95)01057-k.

5. Viswanathan V, Kumpatla S, Tilak P, Kuppusamy A. Relationship between retinal-renal complications among type 2 diabetic subjects in India. Int J Diabetol Vasc Dis Res. 2013;1(2):8-14.

6. Rajalakshmi R, Shanthi Rani CS, Venkatesan U, Unnikrishnan R, Anjana RM, Jeba Rani S, et al. Correlation between markers of renal function and sight-threatening diabetic retinopathy in type 2 diabetes: a longitudinal study in an Indian clinic population. BMJ Open Diabetes Res Care. 2020;8(1):e001325. https://doi.org/10. 1136/bmjdrc-2020-001325.

7. Sabanayagam C, Chee ML, Banu R, et al. Association of diabetic retinopathy and diabetic kidney disease with all-cause and cardiovascular mortality in a multiethnic Asian population. JAMA. 2019;2(3):e191540.

8. Viswanathan V, Krishnamoorthy E, Kumpatla S, et al. Clinical and biochemical characteristics and the association of angiotensin type 1 receptor with normoalbuminuric chronic kidney disease among South Indian Type 2 diabetes population. Int J Diabetes Dev Countries. 2019;39:254-61.

9. Anitharani A and Viswanathan V : Estimated glomerular filtration rate using creatinine based chronic kidney disease epidemiology collaborarion equation. Letter to Editor. Ind J Neprol 2018: 28(6): 492-493.

10. Kumpatla S, Soni A, Viswanathan V. Comparison of two creatinine based equations for routine estimation of GFR in a speciality clinic for diabetes. J Assoc Physicians India. 2017;65(8):38-41.

Publisher's note Springer Nature remains neutral with regard to jurisdictional claims in published maps and institutional affiliations. 